\title{
Practical Exploration of the Third-Party Evaluation of Local Government Target Management

\author{
Gu Yanan ${ }^{a, *}$, Hu Bin ${ }^{b}$
}

(Shanghai University of Engineering Science,Shanghai,China)

aguyanan_888@163.com, bhubinlyj@163.com

Keywords: target management; local government; third-party assessment

\begin{abstract}
The target management of local government operation is an innovation of the traditional government management system and promotes the change of government management from task-oriented to goal-oriented. However, as a typical tool for evaluating the performance of public organizations, relevant theoretical research has lagged behind the practical basis. Therefore, using the case analysis method to carry on the comprehensive research to the construction process of the target management system of the B district government, combing its actual effect and existing problems of the third party assessment of the current target management, the paper puts forward some relevant suggestions: scientific design index system, strengthen the publicity of government information, and improve the relevant legal regulation system. In order to promote the the third party evaluation system of target management at the local level, further enrich the performance assessment system. And then play a certain role of reference and enlightenment to the national scope of radiation.
\end{abstract}

\author{
地方政府目标管理第三方评估的实践探索 \\ 顾亚楠 ${ }^{a, *}$ ，胡斌 $b$ \\ 上海工程技术大学管理学院, 上海, 中国 \\ aguyanan_888@163.com, bhubinlyj@163.com
}

关键词：目标管理；地方政府；第三方评估

中文摘要：地方政府运行目标管理是对传统政府管理体制机制的创新，推动政府管理由任务 导向型向目标导向型的转变。但是, 作为公共组织绩效评估的典型工具, 相关理论研究已严 重滞后于实践基础。为此, 运用案例分析法对 $\mathrm{B}$ 区政府目标管理体系建设过程进行综合研究, 梳理其现行目标管理第三方评估制度的实际成效和存在问题, 并提出相关建议: 科学设计指 标体系、加强政府信息公开、健全相关法律规制体系。以期从地方上先行完善 “目标管理 + 第三方” 的评估制度, 进一步丰富区县级政府绩效评估体系, 进而对全国范围内辐射发挥一 定的借鉴和启示作用。

\section{1.引言}

随着我国行政体制改革的持续推进，政府绩效评估作为国外相关改革经验的有效借鉴日 益受到各级地方政府的重视与推崇。在经验与实际相融合的过程中萌生出了多种独具地方特 色的评估形式，如目标责任制评估、公民评议政府、社会服务承诺制评估、行政效能评估、

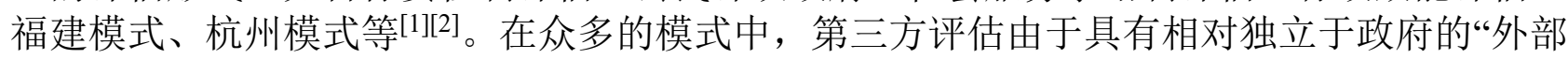
性”特征也逐渐发展成为各地政府绩效管理的重要形式。在一定程度上弥补了政府绩效内部评 
估的不足，极大的提高了政府绩效评价的客观性和公正性。近年来，地方政府不断创新，有 些政府综合运用目标责任制与第三方评估的绩效管理模式也取得不错的成效。但是，由于实 践基础的薄弱, 仍然存在有地方政府人员绩效管理能力欠缺和经验不足的现象。因此, 有必 要对当前绩效评估模式中的问题进行梳理，以期对政府提高行政服务能力有所改善。

文章在前人研究的基础上, 将目标责任制政府与第三方评估相结合讨论, 以上海 B 区政 府目标管理第三方评估为例，分析出在实践中二者相结合的评估模式对政府绩效管理的影响 和存在的问题，并提出了相关的对策建议，从而帮助政府优化行政管理，使绩效评估能够朝 着科学化，规范化和制度化的方向发展。

\section{2.理论基础}

\section{1 目标责任制与政府绩效评估的联系与区别}

当我们假设绩效就是指结果时，政府绩效目标就是指政府目标管理中的目标，而所谓的 目标责任制，实际上就是政府机关在运用目标管理进行绩效评估的过程中将目标管理理论与 岗位责任制相结合的产物。即将组织目标与岗位职责相联系, 通过工作目标设计, 将整体目 标层层分解并逐级下放，最终形成人人担责、权责利明确且相互对称的格局。这种管理模式 以指标体系为中心、以责任体系为根基、以考核体系为动力，已经逐渐成为地方政府有效的 管理实践方式之一。

所谓政府绩效评估，是指政府机关组织或者其他社会组织通过一定的方法、指标和标准， 对政府行政过程中的决策和管理行为在政治、经济、文化、环境等方面所产生的影响和效果 进行分析、比较、评价和测量。其本质上是为了追求政府公共资金受托责任的实现，有助于 权力制衡与权利保障的落实以及责任型政府的实现。它与目标管理的区别在于,政府绩效评估 是以制度设计为依托来建构包含责任、合法性、效率和公正在内的综合性目标体系,以提升公 共服务质量、规范行政行为和提高政府的工作效率。而目标管理则是以对行为人内在的激励 为主, 辅之以相应的制度和责任约束, 旨在催发出内在的行为动力去达成组织和个人的目标。 是一种对人的精神需求提出的高要求，而不是被动的用“鞭子”在身后驱赶。

\section{2 第三方评估的概念和意义}

第三方评估机构相对于政府而言是独立的外部机构，在一定程度上对政府工作起到了监 督和反馈的作用。当前评估模式泛滥, 唯正确的理解和使用绩效评估方能成为政府行政效能 实现的有力推手。那么, 到底什么是第三方评估呢? 学术界也对其赋予了不同的理解。包国宪 等认为, 政府绩效评估可分为内部和外部两种, 内部评估是指由地方政府及其部门自己组织 的对其绩效进行的评估, 而外部评估则是指由与政府机关及其部门无从属和利益关系的第三 方机构组织实施的评估, 其中外部评估通常包括独立第三方评估和委托第三方评估 ${ }^{[3]}$ 。徐双 敏、李跃认为，从国外实行“第三方评估”的经验来看，第三方主要是指处于第一方(被评对象) 和第二方(顾客、服务对象)之外的一方 ${ }^{[4]}$ 。而程样国、李志认为，第三方评估是指由政策制定 者与执行者之外的人员实施的正式评估，主要包括高校专家、专业公司、社会代表、普通民 众四种常见模式 ${ }^{[5]}$ 。

第三方评估模式作为一种必要而有效且无利益牵连的外部制衡机制,在一定程度上弥补 了以往政府内部评估的不足。从社会治理和效率层面来看，第三方评估不仅完善了政府绩效 评估体系,提高了政府绩效评估结果的客观性、公正性和科学性，而且在帮助改善政府整体形 象、增强政府行政能力和民众认同感、加快服务型政府建设等方面都发挥了积极的促进作用。 


\section{B 区政府目标管理第三方评估模式的实践探索}

\section{1“目标管理+第三方评估”的运行机制}

从过去三年的评估经验来看, 绩效评价结果的可靠性和客观性离不开科学评估系统的构 建。课题组在与区政府督查室充分研究后, 设计出了一套较为完整的评估体系来指导绩效评 估工作的进行。第三方评估工作主要从两个维度开展：垂直方向以目标项目划分, 水平方向 以阶段划分（具体如图 1 所示）。

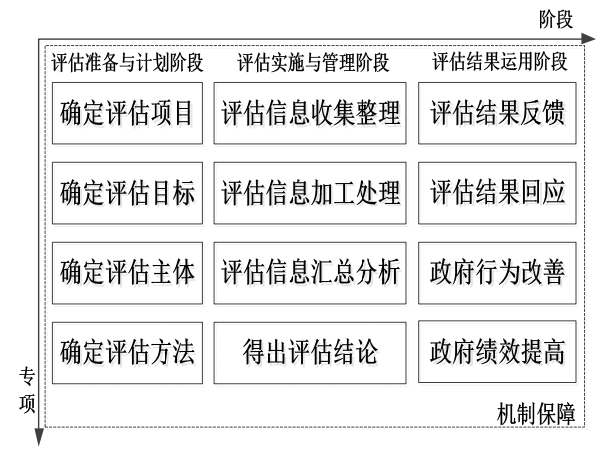

图 1 第三方评估工作维度

\subsection{B 区政府目标管理第三方评估存在的问题}

该区政府虽然已经连续三年进行了目标管理的第三方评估，成效也较为显著，但是由于 起步晚, 实践经验较少, 使得评估管理工作在实际操作过程中依然存在有不少问题, 相关细 节仍需进一步细化和规范。

(1) 评估指标设计不够科学化

在政府绩效评估的过程中，如何建构指标体系是核心问题。科学的指标设置可以为政府 工作指明方向，提供激励。相反，模糊性指标或者愿景性指标过多，可能会增加工作人员的 负担。此次评估中, 多数定性目标缺乏相应的衡量标准, 个别项目甚至还出现与市级项目的 交叉。这就导致权责归属不清, 目标进度滞后。这些组织内生的阻碍大大影响了绩效评估结 果的客观性和科学性, 亟待进一步改善和规范。

(2) 存在主客体之间的信息不对称问题

第三方评估工作得以顺利开展的前提条件就是能够获得评估对象的最新的准确资料。但 是由于政府工作的透明度不高、电子政务不够发达等就会出现评估的主客体之间信息不对称 的问题，往往是报喜不报忧，尤其是定性目标上报时含糊不清，信息不实或者信息更新不到 位都致使第三方工作开展困难，难以判断，个别部门甚至还会存在瞒报谎报现象。虽然课题 组有派遣相关人员全程跟踪，但是体制原因的存在仍然使得信息不对称成为第三方评估有效 推进的一大阻碍。

(3) 缺乏相对完善的法律法规体系

一方面, 区政府自身缺乏有效的法律规制来指导各部门目标管理工作的进行。另一方面, 在协调第三方评估主体与评估对象之间的关系缺乏相应的制度保护。课题组与区政府主要是 以合同的形式来规定双方的权利与义务关系。但是在合同之外的权责关系无法得到保障，进 而导致第三方评估的积极性和有效性降低。

\subsection{B 区政府目标管理第三方评估的启示和建议}

(1) 科学设计指标体系, 减少模糊性愿景性指标

区政府在目标制定过程中应充分考虑与上级部门目标的协调一致，明确权责关系，动态 调整目标, 及时反馈, 避免出现交叉性目标。在指标设置过程中, 可以充分发动上级有关部 门、内部职员代表、外部评估专家以及合作第三方的专业优势，对指标体系提出修改意见， 
尽可能的减少模糊性和愿景性指标的设置, 注重指标设置的质量与群众的接受度，定性指标 与定量指标相结合，设计出相对完善的评估方案。

(2) 加强政府信息公开，大力推行电子政务的发展

政府部门要加快转变思想观念，提高认识，牢固树立政务信息公开的新理念。政务信息 的公开和透明化，有助于社会群体对政府进行全方位的监督，也有助于评估主体充分掌握政 府工作的具体内容和准确信息，获得详尽资料，从而大大提高绩效评估结果的客观性和公正 性。政府部门应该主动适应时代需求，继续深化“互联网+政务”的电子政务模式的实践发展， 实现政务信息的动态管理，对应公开和能公开的信息及时更新和维护，优化政府服务流程。

(3) 进一步健全相关法律规制体系。

基于顶层设计视角审视现行法律体系的不足，建议市政府出台针对性的地方性法律规范 和相关办法，在制度上予以完善，保证政府自身和评估主体能够有法可依，有规可守。以法 制保障第三方评估组织的合法地位和权威，保证其在收集整理资料、开展调研活动、分析绩 效结果、改进评估方案等评价过程的顺利进行。

\section{4. 结语}

实践证明，“目标管理+第三方评估”的绩效评估模式的存在对于地方政府绩效评估发展， 帮助地方政府优化行政效能，促进转型升级具有不可替代的优势。尽管第三方评估在我国起 步较晚, 实践经验比较少, 也出现了许多问题，但是其发挥出的效用是公认的。只不过完善 第三方评估模式还需要一段很长的时间。B 区政府目标管理第三方评估工作的探索，是地方 政府在行政改革方面的积极尝试，比以往政府单一的绩效评估模式收效更好，发现和分析实 践中的问题对于推进地方政府全面推进目标管理工作具有重要意义，对其他地方政府开展类 似的评价活动具有一定的启示性作用。

\section{References}

[1] Chen Xiaohua, Lu Zhipeng. A Comparative Study of Local Government Performance Ev-aluation Models: An Analytical Framework[J]. Economic and Social System Comparison,2019(02):106-116.

[2] Sun Tao, Z Yimeng. Scientific Differentiation Government Performance Evaluation--Tech-nology Governance Tools for Optimizing Government Responsibility System[J]. Journal of National School of Administration, 2018(06):26-31+186.

[3] B.Guoxian, Z Zhidong. Analysis on the Self-discipline of the Third Party Government P-erformance Evaluation Organization in China[J]. Administration Administration of China, 2008(1):49-51.

[4] Xu Shuangmin, Li Yue. The Third Party Evaluation Subject of Government Performance and Its Effect[J]. Chongqing Social Sciences, 2011(9):118-122.

[5] Zhu Xia, L Ying. Current Situation, Problems and Countermeasures of Performance Evaluation of Third-Party Local Governments[J]. Friends of Accounting, 2017(19):115-117. 